\title{
The Brera Multi-scale Wavelet Chandra Survey. I. Serendipitous source catalogue
}

\section{P. Romano*, ${ }^{a}$ S. Campana, ${ }^{b}$ R.P. Mignani, ${ }^{c}$ A. Moretti, ${ }^{b}$ M. Mottini, ${ }^{d}$ M.R. Panzera, ${ }^{b}$ G. Tagliaferri ${ }^{b}$}

a INAF, Istituto di Astrofisica Spaziale e Fisica Cosmica,

Via U. La Malfa 153, I-90146 Palermo, Italy

${ }^{b}$ INAF, Osservatorio Astronomico di Brera,

Via E. Bianchi 46, I-23807 Merate, Italy

${ }^{c}$ Mullard Space Science Laboratory, University College London,

Holmbury St. Mary, Dorking, Surrey, RH5 6NT, UK

${ }^{d}$ European Southern Observatory,

Schwarzschild Straße 2, 85740 Garching bei München, Germany

E-mail: romano@ifc.inaf.it

We present the Brera Multi-scale Wavelet Chandra (BMW-Chandra) source catalogue drawn from essentially all Chandra ACIS-I pointed observations with an exposure time in excess of $10 \mathrm{ks}$ public as of March 2003 (136 observations). Using the wavelet detection algorithm developed by Lazzati et al. (1999) and Campana et al. (1999), which can characterise both point-like and extended sources, we identified 21325 sources. Among them, 16758 are serendipitous, i.e. not associated with the targets of the pointings. This makes our catalogue the largest compilation of Chandra sources to date. The $0.5-10 \mathrm{keV}$ absorption corrected fluxes of these sources range from $\sim 3 \times 10^{-16}$ to $9 \times 10^{-12} \mathrm{erg} \mathrm{cm}^{-2} \mathrm{~s}^{-1}$ with a median of $7 \times 10^{-15} \mathrm{erg} \mathrm{cm}^{-2} \mathrm{~s}^{-1}$. The catalogue consists of count rates and relative errors in three energy bands (total, $0.5-7 \mathrm{keV}$; soft, 0.5-2 keV; and hard, 2-7 keV), where the detection was performed, and source positions relative to the highest signal-to-noise detection among the three bands. The wavelet algorithm also provides an estimate of the extension of the source. We include information drawn from the headers of the original files, as well, and extracted source counts in four additional energy bands, SB1 (0.5-1 keV), SB2 (1-2 keV), HB1 (2-4 keV), and HB2 (4-7 keV). We computed the sky coverage for the full catalogue and for a subset at high Galactic latitude $\left(|b|>20^{\circ}\right)$. Our sky coverage in the soft band ( $0.5-2 \mathrm{keV}, \mathrm{S} / \mathrm{N}=3)$ is $\sim 8 \mathrm{deg}^{2}$ at a limiting flux of $\sim 10^{-13} \mathrm{erg} \mathrm{cm}^{-2} \mathrm{~s}^{-1}$, and $\sim 2$ $\mathrm{deg}^{2}$ at a limiting flux of $\sim 10^{-15} \mathrm{erg} \mathrm{cm}^{-2} \mathrm{~s}^{-1}$.

7th INTEGRAL Workshop

September 8-11 2008

Copenhagen, Denmark

* Speaker. 


\section{Introduction}

The Brera Multi-scale Wavelet (BMW, [4, 1]) algorithm, which was developed to analyse ROSAT High Resolution Imager (HRI) images [6], was modified to support the analysis of Chandra Advanced CCD Imaging Spectrometer (ACIS) images [5], and subsequently led to interesting results on the nature of the cosmic X-ray background [2]. Differently from other WT-based algorithms, the BMW automatically characterises each source through a multi-source $\chi^{2}$ fitting with respect to a Gaussian model in the wavelet space, and has therefore proven to perform well in crowded fields and in conditions of very low background [ $₫$. Given the reliability and versatility of the BMW, we decided to apply it to a large sample of Chandra ACIS-I images, to take full advantage of the superb spatial resolution of Chandra [ 0.5" point-spread function (PSF) on-axis]. We thus produced the Brera Multi-scale Wavelet Chandra Survey [7] and here we present a prerelease of this catalogue, which is based on a subset of the whole Chandra ACIS observations dataset, roughly corresponding to the first three years of operations. Our catalogue provides source positions, count rates, extensions and relative errors.

\section{Method}

\subsection{Sample selection}

We chose the Chandra fields which maximised the sky area not occupied by the pointed targets, that is the fields where the original PI was interested in a single, possibly point-like object centred in the field. Our criteria were the following:

1. All ACIS-I [no grating, no High Resolution Camera (HRC) fields and in Timed Exposure mode] fields with exposure time in excess of $10 \mathrm{ks}$ available by 2003 March were considered. Data from all four front-illuminated (FI) CCDs (I0, I1, I2, I3) were used.

2. We excluded fields dominated by extended sources [covering more than $1 / 9$ of the field of view $(\mathrm{FOV})]$.

3. We excluded planet observations and supernova remnant observations.

4. We also excluded fields with bright point-like or high-surface brightness extended sources.

5. We put no limit on Galactic latitude, but we selected sub-samples based on latitude at a later time.

The exclusion of bright point-like or high-surface brightness extended sources was dictated by the nature of our detection algorithm, which leads to an excessive number of spurious detections at the periphery of the bright source, which is a common problem to most detection algorithms. Therefore, each field was visually inspected to check for such effects (see Figure 1); when found, a conservatively large portion of the field was flagged. Of the 147 fields analysed, $11(\sim 7 \%)$ were discarded because of problems at various stages of the pipeline execution. As a result of our selection, we retained 136 fields. Figure 2 (left) shows the Aitoff projection in Galactic coordinates of their positions. We note that several fields were observed more than once. These fields were considered as different pointings, so that the number of distinct fields is 94 . 

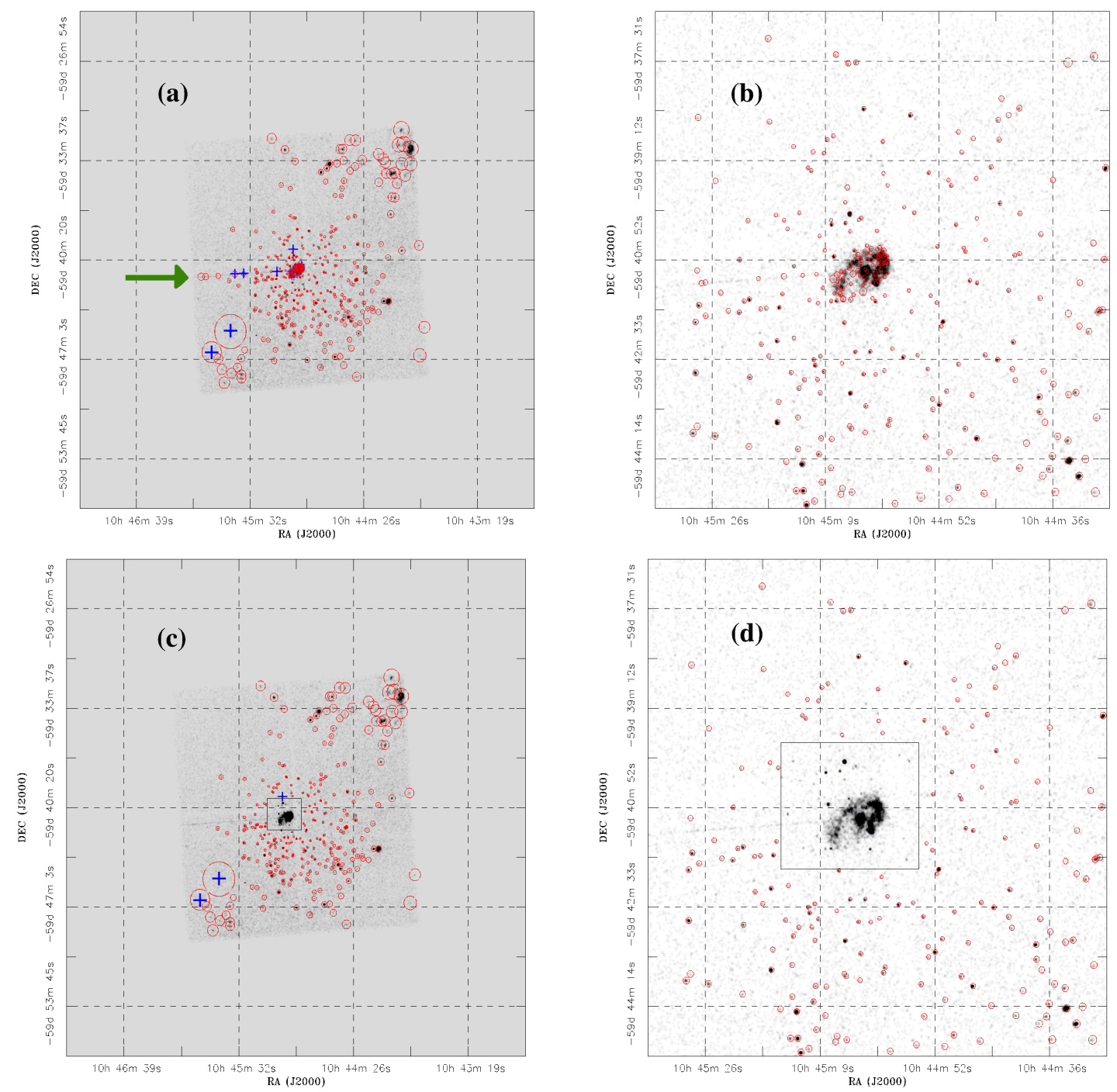

Figure 1: Example of detection. (a) The $\eta$ Carinae full field at half resolution. Note the complicated extended structure at the centre and the spurious detections along a readout streak (green arrow). (b) Central portion of the field at full resolution. Crosses mark sources that the detection algorithm classifies as extended (e.g. left-bottom corner and along readout streak). (c) Example of manual cleaning. The spurious sources along the readout streak were eliminated and (d) the sources in the central portion of the image (contained within the box and not shown) were flagged for later inspection. 
The data input in our pipeline are Level 2 (L2) data generated by the Chandra X-ray Center (CXC) standard data processing in a uniform fashion, which were filtered to only include the standard event grades, and then corrected for aspect offsets. We applied energy filters to these event lists, and created soft (SB, 0.5-2.0 keV), hard ( $\mathrm{HB}, 2.0-7.0 \mathrm{keV})$ and total $(\mathrm{FB}, 0.5-7.0 \mathrm{keV})$ band event files. The upper limit on our hard and total energy bands was chosen at $7 \mathrm{keV}$ because at higher energy the background increases and the effective area decreases, producing lower signalto-noise $(\mathrm{S} / \mathrm{N})$ data. Our results in the $0.5-10 \mathrm{keV}$ band are then extrapolations from our findings in the $0.5-7 \mathrm{keV}$ range.

\subsection{The algorithm}

The main steps of the BMW algorithm can be summarised as follows (full details in [阿; [何; [1]). The first step is the creation of the WT of the input image; the BMW WT is based on the discrete multi-resolution theory and on the "à trous" algorithm, which differs from continuousWT-based algorithms which can sample more scales at the cost of a longer computing time. We used a Mexican hat mother-wavelet, which can be analytically approximated by the difference of two Gaussian functions. The WT decomposes each image into a set of sub-images, each of them carrying the information of the original image at a given scale. This property makes the WT well suited for the analysis of X-ray images, where the scale of sources is not constant over the field of view, because of the dependence of the PSF on the off-axis angle. We used 7 WT scales $a=[1,2,4,8,16,32,64]$ pixels, to cover a wide range of source sizes, where $a$ is the scale of the WT [丹].

Candidate sources are identified as local maxima above the significance threshold in the wavelet space at each scale, so that a list is obtained at each scale, and then a cross-match is performed among the 7 lists to merge them. At the end of this step, we have a first estimate of source positions (the pixel with the highest WT coefficient), source counts (the local maximum of the WT) and a guess of the source extension (the scale at which the WT is maximized). A critical parameter is the detection threshold which, in the context of WT algorithms, is usually fixed arbitrarily by the user in terms of expected spurious detections per field [ [ $]$. The number of expected spurious detections as a function of the threshold value and for each scale was calculated by means of Monte Carlo simulations [5].

The final step is the characterisation of the sources by means of a multi-source $\chi^{2}$ minimization with respect to a Gaussian model source in the WT space. In order to fit the model on a set of independent data, the WT coefficients are decimated according to a scheme described in full in [ 7 ].

We ran the detection algorithm on the source images rebinned by a factor of 2 ( 1 pixel $\sim 0.98$ "), and then in their inner $512 \times 512$ part at the full resolution, using 7 scales. We applied corrections to the source counts for vignetting and PSF modelling (i.e. for using a Gaussian to approximate the PSF function to fit the sources in wavelet space). We excluded the $480 \times 480$ pixel central part in the analysis at rebin 2, then cross-correlated the positions of the sources found at rebin 1 and 2 to exclude common double entries. We repeated this procedure for each of the three energy bands, and cross-correlated the resulting source coordinates to form the definitive list (for coincident sources, the coordinates of the highest $\mathrm{S} / \mathrm{N}$ one were kept). We ran the detection algorithm with a single significance threshold that corresponds to $\sim 0.1$ spurious detections per scale, hence (with 7 scales) $\sim 0.7$ spurious detections per field for each band in which we performed the detection. Given our 
sample of 136 fields, we expect a total of $\sim 95$ spurious sources in the catalogue, or a percentage of $2.7 \%$ (considering the three energy bands, the two images over which the detection was run, and the total number of detected sources, see Sect. 2.3. An example of the results of the detection is shown in Figure 1 .

\subsection{The catalogue}

The wavelet detection produced a catalogue of source positions, count rates, counts, extensions, and relative errors in three bands, as well as the additional information drawn from the headers of the original files for a total of 21325 sources. We also extracted source counts within a box centered around the positions determined with the detection algorithm, with a side which is the $90 \%$ encircled energy diameter at $1.50 \mathrm{keV}$. For the SB, HB, and FB bands the background counts were extracted from the same box from the background image. We extracted source counts in the four additional bands: SB1 $(0.5-1.0 \mathrm{keV})$, SB2 $(1.0-2.0 \mathrm{keV}), \mathrm{HB} 1(2.0-4.0 \mathrm{keV})$, and HB2 (4.0-7.0 keV). We calculated the $0.5-10 \mathrm{keV}$ absorption corrected fluxes by converting the count rates in fluxes assuming a Crab spectrum, i.e. a power law with photon index 2.0, modified with the absorption by Galactic $N_{\mathrm{H}}$ relative to each field; we also provide the $0.5-10 \mathrm{keV}$ observed fluxes (simple Crab spectrum and $N_{\mathrm{H}}=0$ ). The catalogue lists the $0.5-10 \mathrm{keV}$ observed flux, the absorption corrected one and the corresponding conversion factors [see Figure 2 (right)]. Problematic portions (such as extended pointed objects) and pointed objects (within a radius of 30 arcsec from the target position) were flagged.

Figure 3 (left) shows the distribution of the source off-axis angle, which presents a steep increase with collecting area, and a gentler decrease with decreasing sensitivity with off-axis angles. Differently from what found with the BMW-HRI catalogue [6], our distribution does not present a peak at zero off-axis due to pointed sources. To characterise the source extension, which is one of the main features of the WT method, one cannot simply compare the WT width with the instrumental PSF at a given off-axis angle. Thus, we use a $\sigma$-clipping algorithm which divides the distribution of source extensions as a function of off-axis angle in bins of 1' width. The mean and standard deviation are calculated within each bin and all sources which width exceeds $3 \sigma$ the mean value are discarded. The procedure is repeated until convergence is reached. The advantage of this method is that it effectively eliminates truly extended sources, while providing a value for the mean and standard deviation in each bin [4]. The mean value plus the $3 \sigma$ dispersion provides the line discriminating the source extension, but we conservatively classify as extended only the sources that lie $2 \sigma$ above this limit. Combining this threshold with the $3 \sigma$ on the intrinsic dispersion, we obtain a $\sim 4.5 \sigma$ confidence level for the extension classification.

The full catalogue contains 21325 sources, 16834 of which are not associated with bright and/or extended sources, including the pointed ones. Of these, 11124 are detections in the total band, 12631 in the soft, 9775 in the hard band; 4203 sources were only detected in the hard band (see Table 1).

It is particularly important for cosmological studies to have a sample which is not biased toward bright objects. To this end, we constructed the BMW-Chandra Serendipitous Source Catalogue that contains 16758 sources not associated with pointed objects, by excluding sources within a radius of 30 arcsec from the target position. Their sky coverage is shown in Figure 3 (right). 

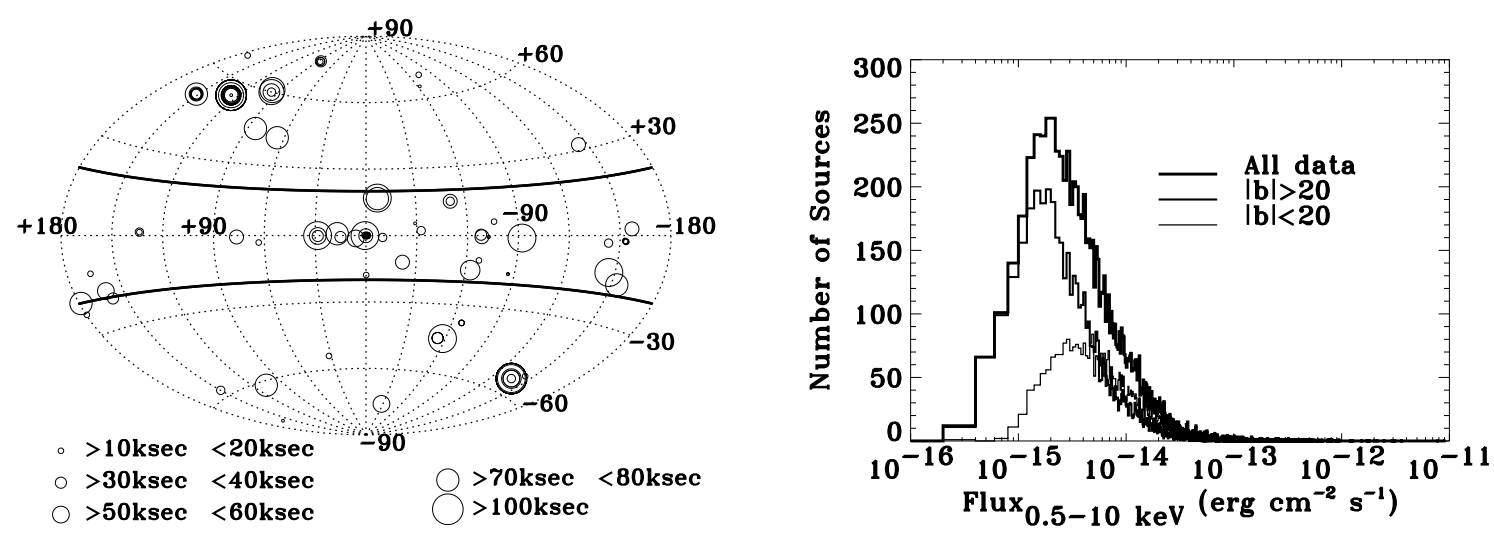

Figure 2: Left: Aitoff Projection in Galactic coordinates of the selected 136 Chandra ACIS-I fields. The thick lines are the limits for the high latitude sub-sample. Right: Distribution of the absorption corrected 0.5-10 keV flux in the full sample, high latitude sample (7401), and low latitude sample (9433).

\section{Catalogue Exploitation}

Among the avenues of scientific exploitation are:

1) Characterization of the sources based on X-ray colours alone;

2) Cross-correlation with other catalogues (FIRST, IRAS, 2MASS, GSC2) allowed the identification of radio-to-optical counterparts; sub-samples of promising sources for optical follow-up include:

i) blank fields (sources without counterparts at other wavelengths; Mignani et al. in prep.);

ii) heavily absorbed sources (the 4203 only detected in the hard X-ray band);

3) Analysis of a sample of 300 extended sources (Fig. 3), which constitutes a list of X-ray selected galaxy cluster candidates, to confirm optically (Romano et al. in prep.);

4) Temporal and spectral variability:

i) autocorrelation of the catalogue allows study of long-term variability of sources observed more than once (Israel et al. in prep.);

ii) intra-observation variability: search for periodicities in the light curves.

\section{BMW-Chandra online}

The current version of the BMW-Chandra source catalogue, (as well as additional information and data) is available at the Brera Observatory and at the INAF-IASF Palermo mirror sites,

http://www.brera.inaf.it/BMC/bmc_home.html

http://www.ifc.inaf.it/ romano/BMC/bmc_home.html

The distributed version can also be found at the Centre de Données astronomiques de Strasbourg (Vizier) and at the HEASARC sites. 

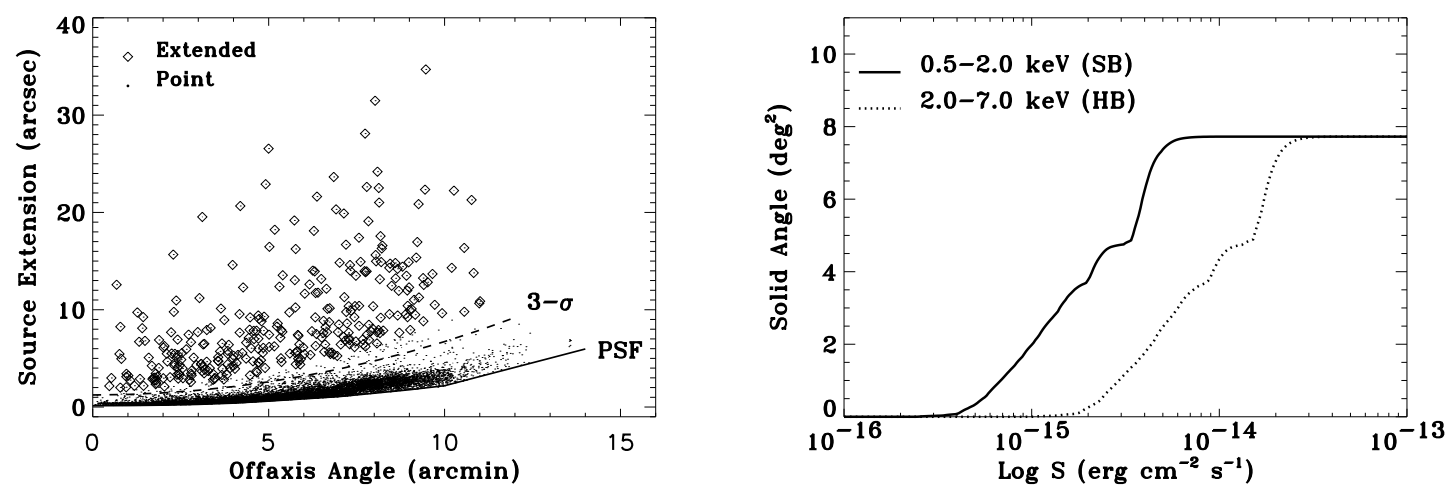

Figure 3: Left: Extension of the BMW Chandra sources as a function of off-axis angle. The solid line is the PSF function, the dashed line is the $3 \sigma$ limit for point sources. Right: Solid angle versus flux limit for $\mathrm{S} / \mathrm{N}=3$ for the soft (solid line) and hard (dotted line) bands. This sky coverage was constructed using 94 independent fields (no fields covered the same sky area).

Table 1: BMW-C in short.

\begin{tabular}{|c|c|c|}
\hline Source Sample & & Number \\
\hline detected & & 21325 \\
\hline $\operatorname{good}^{\mathrm{a}}$ & & 16834 \\
\hline serendipitous & & 16758 \\
\hline \multirow[t]{2}{*}{ independent } & (within 3”) & 12135 \\
\hline & (within 4”.5) & 11954 \\
\hline detected in total band & & 11124 \\
\hline detected in soft band & & 12631 \\
\hline detected in hard band & & 9775 \\
\hline only detected in hard band & & 4203 \\
\hline serendipitous extended & & 316 \\
\hline
\end{tabular}

a Sources which do not require a more in-depth, non-automated analysis (i.e. not associated with bright and/or extended sources at the centre of the field), including the target ones.

\section{Acknowledgments}

This work was supported through Consorzio Nazionale per l'Astronomia e l'Astrofisica (CNAA) and Ministero dell'Istruzione, dell'Università e della Ricerca (MIUR) grants. We thank A. Mistò for his help with the database software. RPM acknowledges STFC for support through its Rolling Grant programme. This publication makes use of data products from the Chandra Data Archive, the FIRST, IRAS, 2MASS, GSC2 surveys. 


\section{References}

[1] Campana, S., Lazzati, D., Panzera, M. R., Tagliaferri, G. 1999, ApJ, 524, 423

[2] Campana, S., Moretti, A., Lazzati, D., Tagliaferri, G. 2001, ApJ, 560, L19

[3] Lazzati, D., Campana, S., Rosati, P., Chincarini, G., Giacconi, R. 1998, A\&A, 331, 41

[4] Lazzati, D., Campana, S., Rosati, P., Panzera, M. R., Tagliaferri, G. 1999, ApJ, 524, 414

[5] Moretti, A., Lazzati, D., Campana, S., Tagliaferri, G. 2002, ApJ, 570, 502

[6] Panzera, M. R., Campana, S., Covino, S., et al., 2003, A\&A, 399, 351

[7] Romano, P., Campana, S., Mignani, R.P., et al., 2008, $A \& A, 488,1221$ 